\title{
AVALIAÇÃO DO ENDOMÉTRIO EM MENOPAUSADAS APÓS USO DE ISOFLAVONAS
}

Luis Paulo Galvão Wolff, Marcos Roberto Martins, Aloísıo José Bedone, llza Maria Urbano Monteiro*

Trabalho realizado na Universidade Estadual de Campinas e Universidade de Taubaté, SP

*Correspondência: R. Timburi,532. Cond. Alphaville

Campinas - SP

ilza@caism.unicamp.br

\begin{abstract}
RESUMO
OBjEtivo. Este estudo descritivo e prospectivo teve como objetivo avaliar o endométrio de mulheres menopausadas antes e após seis meses de uso de isoflavonas totais.

Métodos. Foi realizado um ensaio clínico não controlado, do tipo antes e depois, em 32 mulheres na pós-menopausa, com idade entre 40 e 60 anos, que receberam $80 \mathrm{mg}$ por dia de isoflavonas totais (Trifolium pratense-Climadili ) durante seis meses. Foram avaliadas no início e no final do tratamento por meio de ultra-sonografia pélvica transvaginal, histeroscopia e biópsia endometrial. Os dados coletados foram registrados no programa Epi Info, versão 6,04b e a análise dos dados foi realizada pelo programa estatístico SAS versão 8,2, considerando um nível de significância $(\alpha)$ de 0,05 e um poder (I- $\beta$ ) de 0,80. As variações médias da espessura endometrial, dos achados histeroscópicos e histológicos, entre os instantes iniciais e após seis meses, foram avaliadas pelo teste $t$ de Student para dados pareados.
\end{abstract}

REsultados. Dentre as 32 mulheres que participaram do estudo, seis apresentaram sangramento vağinal e três, alterações endometriais. Duas mulheres desenvolveram proliferação endometrial e uma, hiperplasia endometrial. Não houve alterações significativas em relação ao espessamento endometrial ao ultra-som.

Conclusäo. Neste estudo, três mulheres que utilizaram isoflavonas durante seis meses apresentaram modificações endometriais que sugeriram estímulo endometrial.

UnITERMOS: Isoflavonas. Menopausa. Endométrio. Histeroscopia.

\section{INTRODUÇÃo}

A menopausa é um evento natural que ocorre em mulheres em torno dos 50 anos, sendo decorrente da falência gonadal e caracterizada por deficiência de hormônios esteróides. O hipoestrogenismo pode causar diversos distúrbios como sintomas vasomotores, doenças cardiovasculares, osteoporose, alterações urogenitais e distúrbios cognitivos ${ }^{1,2}$. O uso de estrógenos e progestógenos tornou-se bastante difundido nas últimas décadas'. Tal prática, no entanto, pode ocasionar efeitos adversos como mastalgias, sangramentos uterinos irregulares e aumento do risco relativo para neoplasias de mama ${ }^{3,4,5}$ e endométrio ${ }^{6}$.

O receio das mulheres em relação às neoplasias, além das já conhecidas contra-indicações e intolerâncias medicamentosas, tem aumentado a não aceitação do tratamento hormonal. Os clínicos têm oferecido alternativas terapêuticas com substâncias provenientes da extração de plantas e que podem apresentar efeito estrogênico, denominadas fitoestrógenos ${ }^{7}$. Os fitoestrógenos são classificados em quatro grandes grupos: esteróis, terpenóides, saponinas e fenólicos. Dentro do grupo fenólico, encontramos as isoflavonas, lignanos, coumestanos, flavanóis, favonas, chalconas e os esteróis ${ }^{8}$. As isoflavonas apresentamse entre as classes que possuem maior atividade estrogênica e são encontradas em vários vegetais e produtos manufaturados. Dentre as isoflavonas, os principais compostos são formados por genisteína, dadzeína, biochanina A e formononetina $a^{9,10,11}$.

Estas substâncias são absorvidas no intestino, com metabolização hepática e excreção, principalmente renal. Pequena parte destas substâncias é eliminada pela vesícula biliar e intestino ${ }^{12,8}$.
As ações das isoflavonas nos tecidos alvos parecem ocorrer por meio de dois mecanismos classificados como genômico e não genômico. Os efeitos expressos pelo mecanismo não genômico são determinados por ações sobre a proliferação celular, inibição enzimática, inibição da angiogênese e efeitos antioxidantes ${ }^{13,14}$. Também são descritas ações de inibição da expressão e transcrição de alguns genes que promovem a regulação da proliferação, diferenciação e apoptose celular ${ }^{14}$.

O mecanismo genômico, determinado pela ligação com receptores nucleares específicos, resulta em efeitos estrogênicos ou antiestrogênicos ${ }^{12}$. Os receptores estrogênicos são de dois tipos: $\alpha$ (RE $\alpha$ ) e $\beta(R E \beta)^{15}$. A concentração dos receptores nos tecidos determina sua resposta ao hormônio ${ }^{16}$. Estudos sugerem que as distribuições destes receptores nos tecidos variam, sendo que os RE $\alpha$ apresentam uma larga expressão tecidual principalmente nos tecidos endometriais ${ }^{17}$, mamários e hepáticos ${ }^{8}$. Os $\beta$, no entanto, possuem uma expressão mais focal, com altos níveis principalmente em ovário, próstata, epidídimo, pulmão, hipotálamo e vasos sangüíneos ${ }^{8,18}$.

A seletividade dos fitoestrógenos para os diferentes receptores também é importante na modulação da resposta tecidual. As isoflavonas apresentam maior afinidade para receptores $\beta^{18,19}$, que são pouco expressivos nos tecidos endometriais e mamários, o que determinaria pequena ou nenhuma ação nestes locais. Entretanto, em alguns estudos, observou-se que as isoflavonas apresentaram efeito estrogênico nos tecidos endometrial e mamário ${ }^{17,12,18,20,21}$. 
Outra variável importante é a concentração da droga. Alguns fitoestrógenos podem exercer efeito agonista ou antagonista, dependendo de sua concentração 20 .

As isoflavonas, portanto, podem apresentar efeito estrogênico ou antiestrogênico, dependendo do tipo de receptor estimulado, da concentração destes receptores no tecido, do tipo de isoflavona e de sua concentração no organismo.

Estas variáveis envolvidas com o mecanismo de ação das isoflavonas podem explicar as controvérsias observadas nos estudos. Ainda existem dúvidas sobre o efeito endometrial com o uso prolongado das isoflavonas em mulheres após a menopausa. Uma das preocupações existentes é o possível estímulo e conseqüente proliferação endometrial, que poderia determinar o aparecimento da hiperplasia endometrial, o precursor mais comum do carcinoma de endométrio ${ }^{22}$.

Uma melhor avaliação da resposta endometrial ao uso das isoflavonas permitirá melhor orientação quanto aos riscos e benefícios do uso deste medicamento e da necessidade ou não da realização de proteção endometrial.

\section{Métodos}

Foi realizado um estudo do tipo descritivo e prospectivo para o qual foram selecionadas 55 mulheres menopausadas com queixas de fogacho, que procuraram os serviços de climatério do Hospital Universitário de Taubaté (HUT) ou o serviço de climatério da Prefeitura Municipal de Pindamonhangaba (PMP). As mulheres eleitas para este estudo não tinham realizado qualquer tipo de tratamento hormonal nos últimos seis meses, apresentavam idade entre 40 e 60 anos, tempo de menopausa inferior a cinco anos e dosagem sérica de FSH superior a $30 \mathrm{U} / \mathrm{L}$. Mulheres com história de neoplásica maligna ginecológica, antecedentes pessoais de tireioidopatias ou osteoporose, alteração endometrial sugestiva de processo proliferativo ou na impossibilidade de realização da propedêutica endometrial foram excluídas do estudo. Foi realizado um ensaio clínico não controlado do tipo antes e depois, em que todos os sujeitos receberam comprimidos contendo isoflavonas totais na quantidade de $230 \mathrm{mg}$ de extrato seco de Trifolium pratense L. $\left(\right.$ Climadi $\left.^{\circledR}\right)$, na posologia de $80 \mathrm{mg}$ por dia, e foram comparados quanto aos achados endometriais no início e após seis meses de seguimento, com o objetivo de se avaliar a resposta endometrial. A avaliação endometrial foi obtida por meio do exame ultra-sonográfico transvaginal, histeroscopia diagnóstica e biópsia orientada do estudo.

O exame ultra-sonográfico foi realizado por via transvaginal (transdutor de 7,5 mHz), em aparelho modelo Sonoace 8800 ou Aloka SSD 500, sendo a espessura endometrial avaliada em corte longitudinal, incluindo as medidas das camadas basais. Considerou-se alterada a espessura endometrial superior a $5 \mathrm{~mm}^{24,25}$ no exame inicial ou com diferença superior a Imm, na avaliação aos seis meses ${ }^{26}$. A histeroscopia diagnóstica foi realizada ambulatorialmente, com óptica Endoview de 4,0 mm e distensão da cavidade uterina com CO2. O exame foi classificado em normal (endométrio atrófico ou hipotrófico), hipertrofia endometrial, sugestivo de hiperplasia ou neoplasia endometrial e outros (pólipos, miomas e sinéquias). Após a histeroscopia, foi realizada biópsia aspirativa com Pipelle ${ }^{\circledR}$ e o material foi fixado em formol a $10 \%$ e corado com hematolina-eosina e o laudo emitido pelo patologista respeitou a classificação histológica de Kurman e Norris de 198727. Foram calculadas as médias, medianas e desvio padrão das variáveis estudadas e aplicados os testes t de Student para dados pareados e de Wilcoxon para amostras dependentes. Todas as mulheres selecionadas para o estudo assinaram o consentimento livre e esclarecido após terem sido orientadas a respeito da pesquisa. Este estudo obteve aprovação do Comitê de Ética em Pesquisa da Faculdade de Ciências Médicas da Universidade Estadual de Campinas (Unicamp) tendo sido cumpridos os princípios enunciados na Declaração de Helsinki².

Dentre as 55 mulheres selecionadas, 22 foram excluídas. Os motivos da exclusão foram a presença de alterações ultra-sonográicas ou histeroscópicas no exame inicial, dificuldade na progressão da óptica por estenose de canal cervical ou por não retornarem para o início do tratamento. Uma das eleitas não concluiu o estudo. Dessa forma, 32 mulheres terminaram o estudo.

\section{Resultados}

A idade média das pacientes foi 50,8 anos ( $D P=4,5)$, o tempo de menopausa médio foi de 29,4 meses (variação de 12 a 60 meses). 0 período sem tratamento hormonal variou de 6 a 60 meses, com um tempo médio de 18,6 meses. O índice de massa corpórea (IMC) médio no início de foi de $21,8 \mathrm{~kg} / \mathrm{m}^{2}$ e a mediana, $20,8 \mathrm{~kg} / \mathrm{m}^{2}$, dentro da faixa de peso adequado.

O exame ultra-sonográfico transvaginal não demonstrou alterações significativas tanto para a espessura endometrial quanto para o volume uterino, comparando-se os resultados antes e após os seis meses de isoflavona (Tabela I).

Em relação aos achados histeroscópicos e histológicos, três mulheres apresentaram alterações endometriais na avaliação final do estudo. Duas destas mulheres apresentaram endométrio com características proliferativas na histeroscopia e na histologia e uma com alterações sugestivas de hiperplasia simples sem atipias na histeroscopia e na histologia.

Durante o estudo, seis mulheres apresentaram sangramento genital espontâneo, que cessou após alguns dias. Destas, duas apresentaram alterações endometriais ao final do estudo, sendo uma com endométrio proliferativo e outra com endométrio hiperplásico. As outras quatro mulheres não tiveram qualquer alteração endometrial diagnosticada. Na Tabela 2, estão as características das mulheres que apresentaram sangramento genital.

Uma das mulheres do estudo apresentou alteração endometrial, porém sem sangramento genital. A Tabela 3 mostra as características das três mulheres que apresentaram alterações endometriais.

\section{Discussão}

Neste estudo, observamos que três mulheres que utilizaram isoflavonas durante seis meses apresentaram algum tipo de mudança na histologia endometrial. Estas mulheres, com propedêutica endometrial inicial compatível com endométrio inativo, desenvolveram proliferação endometrial (duas) e, até 


\begin{tabular}{|c|c|c|c|c|c|c|c|}
\hline Ultra-som & $n$ & média & DP & mínimo & máximo & mediana & $p$ \\
\hline LE inicial & 32 & 3,4 & $\overline{1,0}$ & $\overline{1,0}$ & $\overline{5,0}$ & $\overline{3,0}$ & 0,2604 \\
\hline LE final & 32 & 3,6 & 1,1 & 2,0 & 6,0 & 4,0 & \\
\hline Vol inicial & 32 & 56,5 & 29,5 & 23,0 & 163,0 & 50,0 & 0,0598 \\
\hline Vol final & 32 & 51,7 & 22,8 & 24,0 & 136,0 & 48,0 & \\
\hline
\end{tabular}

Teste de Wilcoxon para amostras dependentes $\quad \mathrm{DP}=$ desvio-padrão

Tabela 2 - Correlação entre idade, tempo de menopausa, IMC* e achados endometriais das mulheres que apresentaram sangramento genital

\begin{tabular}{|c|c|c|c|c|c|c|c|}
\hline \multirow[b]{2}{*}{ Caso } & \multirow[b]{2}{*}{ Idade } & \multirow[b]{2}{*}{ Tempo de Menopausa } & \multirow[b]{2}{*}{ IMC } & \multicolumn{2}{|c|}{ Espessura endometrial } & \multicolumn{2}{|c|}{ Achados endometriais } \\
\hline & & & & Inicial & Final & Histeroscopia & Histologia \\
\hline 4 & 52 & 60 & 19,9 & 4 & 3 & Normal & Atrófico \\
\hline 6 & 41 & 24 & 18,7 & 3 & 3 & Espessado & Hiperplasia \\
\hline$\|$ & 53 & 36 & 20,3 & 4 & 4 & Normal & Atrófico \\
\hline 12 & 54 & 18 & 16,0 & 3 & 2 & Normal & Atrófico \\
\hline 15 & 44 & 60 & 24,1 & 4 & 5 & Normal & Atrófico \\
\hline 27 & 43 & 12 & 28,9 & 4 & 4 & Espessado & Proliferativo \\
\hline
\end{tabular}

*IMC = Índice de massa corpórea inicial

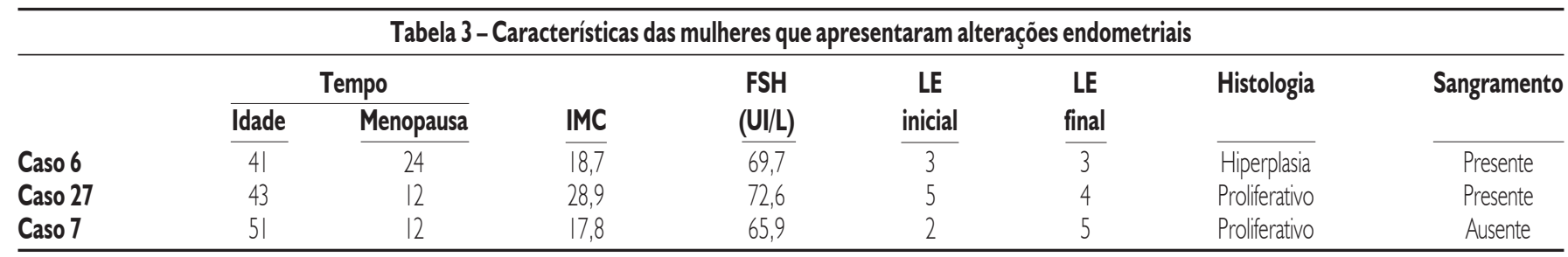

IMC = IMC inicial

mesmo, hiperplasia endometrial simples (uma). As alterações encontradas no presente estudo sugerem que o uso de $80 \mathrm{mg}$ de isoflavonas pode promover um estímulo proliferativo no endométrio em decorrência de uma ação estrogênica agonista. Em um dos casos, a persistência deste estímulo parece ter determinado o desenvolvimento de hiperplasia endometrial.

Não é possível afirmar categoricamente que existe uma relação de causa e efeito entre os achados endometriais e o uso de isoflavona neste estudo, mesmo porque outros fatores poderiam ter influenciado. Entretanto, chama a atenção o fato de que a droga foi administrada por apenas seis meses e que estas pacientes apresentavam uma propedêutica endometrial completa normal no início do estudo. Do mesmo modo, não foram identificados fatores que justificassem estas alterações; nos três casos, as mulheres não eram obesas e a menopausa tinha ocorrido havia mais de um ano.

As alterações por nós observadas são corroboradas por outro estudo em que foram acompanhadas 179 mulheres em uso de $150 \mathrm{mg}$ de isoflavonas durante cinco anos, no qual se observou o aparecimento de seis casos de hiperplasia endometrial e cinco casos de endométrio proliferativo, totalizando II casos (7\%) de alterações endometriais. Sugere-se que estes resultados sejam decorrentes da ação estrogênica agonista, mais evidente em tratamentos prolongados, que, quando não adequadamente contrabalanceada pela administração de progesterona, poderia aumentar o risco de hiperplasia endometria|28.
Na maioria dos estudos, a avaliação endometrial é realizada exclusivamente por meio do exame ultra-sonográfico ${ }^{30,31,32}$. A sensibilidade e especificidade deste método são definidas por sua capacidade de rastrear o câncer do endométrio ${ }^{33}$. Em nosso estudo, o exame ultrasonográfico não detectou as alterações endometriais observadas por meio da histeroscopia e da histologia. É possível que seja necessário mais tempo para que o exame ultra-sonográfico detecte alterações endometriais, que se baseiam principalmente no aumento da espessura endometrial.

Também não observamos estrita correlação entre sangramento genital e estímulo endometrial. Dentre as três mulheres com alterações histológicas, uma não apresentou sangramento genital. Por outro lado, quatro mulheres com sangramento não mostraram alterações endometriais.

Estas alterações endometriais poderiam ser causadas pelo mecanismo genômico relacionado aos receptores endometriais. $\mathrm{O}$ aparecimento de um caso de hiperplasia complexa em estudo já citado ${ }^{28}$ sugere que as ações relacionadas ao mecanismo não genômico, com efeitos principalmente sobre a proliferação celular e inibição enzimática, não são as mais eficazes. Isto pode ser verificado em estudos em que se observou que a administração de isoflavonas não promoveu inibição no crescimento celular de alguns tipos histológicos de neoplasias de mama ${ }^{7,18}$ e células tumorais endometriais ${ }^{34}$. Em outro estudo em que se analisou por meio da imunoistoquímica o antígeno de 
proliferação celular Ki-67 em mulheres na perimenopausa, não foi evidenciado que as isoflavonas pudessem promover qualquer efeito antiproliferativo endometria ${ }^{35}$.

Ao que parece, a ação das isoflavonas deve estar mais relacionada ao mecanismo genômico, diretamente influenciado pelas respostas hormonais e decorrente de receptores estrogênicos ${ }^{12}$. O efeito estrogênico promovido pelas isoflavonas já demonstrado em diversos estudos ${ }^{36,7,72,8}$ é preocupante em relação à sua capacidade de promover estimulação dos diversos órgãos e tecidos. Vários autores demonstraram que o efeito estrogênico é decorrente da ligação a receptores específicos localizados no órgão-alvo ${ }^{16,37,17}$ e que fatores como número de receptores ${ }^{16}$, tipo de receptores ${ }^{15}$, bem como o tempo de exposição e o momento do início da exposição são importantes para definição da resposta ao estímulo hormonal promovido pelas isoflavonas ${ }^{7,38,20}$.

É provável que, com a falta de estrogênio, os receptores endometriais livres liguem-se com mais afinidade à isoflavona. Apesar disso, deve-se lembrar que no endométrio predominam os RE $\alpha$ e as isoflavonas ligam-se prefencialmente com $\operatorname{RE} \beta^{17,18,19}$, o que ocasiona um efeito estrogênico fraco ${ }^{7} \mathrm{com}$ menor estímulo endometrial ${ }^{12,20}$.

\section{Conclusão}

Dessa forma, considerando-se na menopausa níveis residuais de estrogênio sérico ${ }^{29}$, a possibilidade da presença no endométrio de $R E \alpha$, e ligação das isoflavonas a estes receptores e também a $R E \beta^{17,18,19}$, e considerando-se o tempo de exposição com formação de novos receptores endometriais estimulados pelo efeito estrogênico prolongado ${ }^{16}$, é possível que a utilização de isoflavonas por um período longo promova alterações endometriais ${ }^{28}$. Assim, a preocupação com 0 estímulo endometrial quanto à utilização de isoflavonas por um período prolongado parece ser uma atitude prudente. Diante destas constatações, são necessários mais estudos com maior número de pacientes acompanhadas durante um tempo maior, assim como análises imunoistoquímicas e de biologia molecular para maior compreensão da ação das isoflavonas sobre o endométrio.

\section{Conflito de interesse: não há.}

\section{SUMMARY}

\section{Endometrial eVAluation in menopausal WOMEN AFTER SIX MONTHS OF ISOFLAVONES}

OBJETINE. To evaluate the endometrium of menopausa/women before and after six months use of total isoflavone.

METHODS. A non-controlled clinical, type "before and after" trial was carried out with 32 post-menopause women, between 40 and 60 years of age. They received $80 \mathrm{mg} /$ day of total isoflavones obtained from Trifolium pratense (Climadilâ) during six months. They were evaluated at the beginning and end of treatment by transvaginal pelvic ecography, hysteroscopy and endometrial biopsy.

DATAANALYSIS. Data collected were registered by means of the Epi info, version $6.04 \mathrm{~b}$ software, data analysis was made using the SAS version 8.2 statistics program, considering a significance level $(\alpha)$ of 0.05 and a 0.80 power (I- $\beta)$. In order to study the average variation of endometrial thickness and variation of the hysteroscopic and hystologic findings the $t$ Student test for paired data was used.
RESULTS. Among the 32 participant women, six presented vaginal bleeding and three presented endometrial alteration when compared to the initial exams. Two of the women developed endometrial cell proliferation and one of them endometrial hiperplasia. There were no significant alterations in relation to endometrial thickness.

CONCLUSION. In this study, three women who used isoflavones during the six month period presented endometrial activity. [Rev Assoc Med Bras 2006; 52(6): 419-23]

KEY WORDS: Isoflavones. Menopause. Endometrium. Hysteroscopy.

\section{REFERÊNCIAS}

I. Ferreira JAS. A perimenopausa. In: Fernandes CE, Melo NR, Wheba S. Climatério feminino. São Paulo: Ed. Lemos; 1999. p.4I-56.

2. Navarro PAAS, Gomes FM, Azevedo GD, Costa FS, Ferriani RA, lannetta O. Fatores locais envolvidos na etiopatogênese da osteoporose pósmenopausa. Reprod Clim. 2001 ; 16: 167-72.

3. Colditz GA. Relationship between estrogen levels: use of hormone replacement therapy and breast cancer. J Nat Cancer Inst. 1998;90:8|4-23.

4. Garnet AL, Howard JL, Kaunitz AM, Andrew M, Barad DH, Beresford SAA, et al. Effects of estrogen plus progestin on gynecologic cancers and associated diagnostic procedures: The Women's Health Initiative Randomized Trial. JAMA. 2003;290: 1739-48.

5. Hays J, Ockene JK, Brunner RL, Kotchen JM, Manson JE, Patterson RE, et al. Effects of estrogen plus progestin on health-related quality of life. N Engl J Med. 2003;348: I 839-54.

6. American Cancer Society. American cancer statistics 2004 [online]. Atlanta; 2004. Avaliable from: http://www.cancer.org/downloads/STT/ CAF_finalPWSecured.pvx $>$ [cited nov 2004].

7. Allred CD, Allred KF, JuYH, Virant SM, Helferich WG. Soy diets containing varying amounts of genistein stimulate growth of estrogen-dependent (MCF-7) tumors in a dose-dependent manner. Cancer Res. 2001;61:5045-50.

8. Alves DL, Silva CR. Fitohormônios: abordagem natural da terapia hormonal. São Paulo: Atheneu; 2002. p. I-54.

9. Nachtigall LE. Isoflavones in the management of menopause. J Br Menopause Soc. 200 I;(Suppl I):8-I I.

10. Wender, M.C.; Campos, L.S. Fitoestrogênios: examinando as evidências do seu emprego no climatério. Reprod Clim. 2001; | 6: I 55-62.

II. Lethaby AE, Konenberg F, Roberts H, Eden J. Phytoestrogens for menopausal symptoms (Protocol for a Cochrane Review). Cochrane Library; I (I) [online]. Avaliable from: http://www.bireme.br/cgi-bin/ wxislind.exe/iah-cochrany. [cited maio 2002]

12. Dornstauder E, Jisa E, Unterrieder I, Krenn, L, Kubelka W, Jungbauer A. Estrogenic activity of two standardized red clover extracts (MenoflavonÒ) intended for large scale use in hormone replacement therapy. J Steroid Biochem Mol Biol. $2001 ; 78: 67-75$.

13. Boersma BJ, Barnes S, Kirk M, Wang CC, Smith M, Kim H, et al. Soy isoflavonoids and cancer metabolism at the target site. Mutat Res. $2001 ; 480-481: 121-7$

14. Lian Z, Niwa K, Tagami K, Hashimoto M, Gao, J, Yokoyama Y, et al. Preventive effects of isoflavones, genistein and daidzein, on estradiol- I 7brelated endometrial carcinogenesis in mice. Jpn J Cancer Res. 2001;92:726-34.

15. Couse JF, Lindzeu J, Grandien K, Gustafsson JÁ, Korach KS. Tissue distribution and quantitative analysis of estrogen receptor a (ERa) and estrogen receptor $b(E R b)$ messenger ribonucleic acid in the wild-type and ERa-Knockout mouse. Endocrinology. 1997; 138:4613-21.

16. Lipsett MB. Hormônios esteróides. In: Yen SSC, Jaffe RB, Endocrinologia reprodutiva: fisiologia, fisiopatologia e tratamento clínico. $2^{a}$ ed. São Paulo: Ed. Roca; 1999. p. I35-47. 
17. Utsunomiya H, Suzuki T, Harada N, Kiyoshi I, Matsuzaki S, Konnor R, et al. Analysis of estrogen receptor $\mathrm{a}$ and $\mathrm{b}$ in endometrial carcinomas: correlation with ERb and clinicopathologic findings in 45 cases. Int J Gynecol Pathol. 2000; 19:335-4I.

18. Morito K, Hirose T, Kinjo J, Hirakawa T, Okawa M, Nohara T. Interaction of phytoestrogens with estrogen receptors a and b. Biol Pharm Bull $2001 ; 24: 351-6$.

19. Urbanetz AA, Ribeiro MC. Fitoestrogênios: verdade ou mito? Reprod Clim.200I; | 6:92-7.

20. Mueller SO, Simon S, Chac K, Metzier M, Korach KS. Phytoestrogens and their human metabolites show distinct agonistic and antagonistic properties on estrogen receptor a (ERa) and b (ERb) in human cells. Toxicol Sci. 2004;80: I 4-25.

21. Piovesan AC, Simões RS, Mosquete R, Simões Junior MJ, Baracat EC. Efeitos das isoflavonas no tecido mamário. Femina. 2004;32:759-63.

22. Montgomery BE, Daum GS, Dunton C]. Endometrial hyperplasia: a review. Obstet Gynecol Surv. 2004;59:368-78.

23. Associação Médica Mundial. Declaração de Helsinki: princípios éticos para as investigações médicas em seres humanos [online]. Revisada na 52a assembléia geral, Edinburgo; 2000. Disponível em: http://wma.net/e/ policy/b3.htm. [citado nov 2004].

24. Bourne T, Hamberger L, Hahlin M, Granberg S. Ultrasound in gynecology: endometrium. Int J Gynecol Obstet. 1997;56: I I 5-27.

25. Moura AP, Pastore AR, Cerri GG. Endométrio. In: Pastore AR, Cerri GG. Ultra-sonografia: obstetrícia-ginecologia. São Paulo: Sarvier; 1997. p.555-7I.

26. Epstein E, Valentin L. Intraobserver and interobserver reproducibility of ultrasound measurements of endometrial thickness in postmenopausal women. Ultrasound Obstet Gynecol. 2002;20:486-91.

27. Kurman RJ, Norris HJ. Endometrial hyperplasia and metaplasia. In: Kurman RJ. Blaustein's pathology of the female genital tract. $3^{\text {a }}$ ed. New York: Ed. Springer-Verlag; 1987. p.322-37.

28. Unfer V, Casini ML, Costabile L, Mignosa M, Gerli S, Di Renzo GC. Endometrial effects of long-term treatment with phytoestrogens: a randomized, double-blind, placebo-controlled study. Fertil Steril. 2004;82: |45-8.

29. Grodin JM, Stiiteri PK, Mac-Donald PC. Source of estrogen production in post-menopausal women. J Clin Endocrinol Metab. 1973;36:207- I 8.
30. Baber RJ, Templeman C, Morton T, Kelly GE, West L. Randomized placebo-controlled trial of an isoflavone supplement and menopausal symptons in women. Climateric. 1999;2:85-92.

31. Han KK, Soares Junior JM, Haidar MA, Lima GR, Baracat EC. Benefits of soy isoflavone therapeutic regimen on menopausal synptoms. Am Col Obstet Gynecol 2002; 99:389-94

32. Penotti M, Fabio E, Modena BA, Rinaldi M, Omodei U, Vigano P. Effect of soy-derived isoflavones on hot flushes, endometrial thickness, and the pulsatility index of the uterine and cerebral arteries. Fertil Steril. 2003;79: I | 12-7

33. Symonds I. Ultrasound, hysteroscopy and endometrial biopsy in the investigation of endometrial cancer. Best Pract Res Clin Obstet Gynaecol. $2001 ;|5: 38|-9 \mid$.

34. Diel P, Smolnikar K, Schultz T, Laudenbach-Leschowski U, Michna $H$, Vollmer G. Phytoestrogens and carcinogenesis - differential effects of genistein in experimental models of normal and malignant rat endometrium. Hum Reprod. 2001 ; 16:997-1006.

35. Hale GE, Hughes CL, Robboy SJ, Argawal SK, Bievre M. A double-blind randomized study on the effects of red clover isoflavone on the endometrium. Menopause. 2001;8:338-46.

36. Bennets HW, Underwood EJ, Shier FL. A specific breeding problem of sheep on subterranean clover pastures in Western Australia. Aust Vet J. 1946;22:2-12.

37. Speroff L. The estrogen receptor: changing conceps. Clinical lessons from molecular biology. In: Coutinho EM, Spinola P. Reproductive medicine: a millennium review. New York: The Parthenon Publishing Group; 1999. p. $|55-6|$.

Artigo recebido: 17/01/06

Aceito para publicação: 19/06/06 